\title{
Cytogenetic mapping of the ALMT (aluminum-activated malate transporter) gene in
}

\section{wheat genotypes}

\author{
Érica Cristina de Oliveira*๑๐, Cecília Alzira Ferreira Pinto-Maglio®
}

Instituto Agronômico/Centro de Recursos Genéticos Vegetais, Av. Barão de Itapura, 1481 - 13020-902 Campinas, SP - Brasil.

*Corresponding author <ericanice4@gmail.com>

Edited by: Leonardo Oliveira Medici

Received January 18, 2019

Accepted April 02, 2019

\begin{abstract}
Presence of the ALMT1 (aluminum-activated malate transporter) gene confers resistance to aluminum toxicity in Triticum aestivum (common wheat). No resistant cultivars of Triticum turgidum ssp. Durum Desf. (durum wheat) have been registered in Brazil. The aim of this study was to map the ALMT1 through application of the FISH (fluorescence in situ hybridization) technique in five wheat genotypes, common and durum, from the Active Germplasm Bank (AGB) of the Instituto Agronômico (IAC): BH 1146, P19, P33, Anahuac, and IAC 1003. FISH-ALMT1 signals were registered in Anahuac (sensitive) chromosomes and in BH 1146, P19, and P33 (resistant) chromosomes. In the three resistant genotypes, a characteristic double FISH signal was found, located in different chromosomes of the complements: in BH 1146 in chromosome 5D, in P19 in 3B, and in P33 in 6B. This FISH - ALMT1 mapping allows for introgression of aluminum resistance in sensitive cultivars through breeding programs using introgression lines containing these carrier chromosomes.
\end{abstract}

Keywords: Triticum sp., molecular cytogenetics, fluorescence in situ hybridization, aluminum tolerance, toxicity

\section{Introduction}

In Brazil, growing durum wheat, as opposed to common wheat, runs into limitations because of its sensitivity to acidic soil, in which there is a high concentration of $\mathrm{Al}^{3+}$ and $\mathrm{H}^{+}$ions (Camargo et al., 2006; Del-Guercio and Camargo, 2011). Studies have been conducted for the purpose of making large-scale growing of durum wheat feasible in the southeastern region of Brazil, especially in the state of São Paulo (Camargo et al., 1999; Camargo et al., 2006; Del-Guercio and Camargo, 2011).

A number of genes related to aluminum toxicity have already been identified in common wheat belonging to different categories, including the ALMT (aluminum-activated malate transporter) family (Raman et al., 2005; Inostroza-Blancheteau et al., 2010; Riaz et al., 2018).

The first gene cloned from the ALMT family was TaALMT1 (Triticum aestivum ALMT1), that codifies the transport protein involved in exudation of malate by the root tip (Sasaki et al., 2004). The common wheat cultivar BH 1146 has a gene that confers resistance to aluminum toxicity denominated AltBH (Riede and Anderson, 1996). According to Raman et al. (2005), the AltBH gene may correspond to the ALMT1 cloned by Sasaki et al. (2004). The ALMT1 was genetically mapped by Luo and Dvorak (1996), Raman et al. (2005) and Soto-Cerda et al. (2015) and indicated their location in the 4D chromosome of common wheat. Raman et al. (2005) also determined the structure of the ALMT1, which consists of six exons and five introns, a 1388 base pairs (bp) coding region, and the total gene with $3968 \mathrm{bp}$, including the non-coding region.

Cytogenetic mapping through the fluorescence in situ hybridization (FISH) technique of nucleic acids is a tool that allows specific DNA and gene sequences to be directly found within the chromosomal complement (Pedrosa et al., 2002; Lukaszewski et al., 2012; Zhang et al., 2013; Tang et al., 2014; Zhang et al., 2015). As of today, there is no record of studies featuring the mapping of the ALMT gene using the FISH technique either in common wheat or in durum wheat. For the latter, there is no record of either genetic mapping or mapping through FISH. The aim of this study was to cytogenetically map the ALMT1 gene in common wheat and durum wheat, using the fluorescence in situ hybridization technique.

\section{Materials and Methods}

\section{Plant material}

Five genotypes were used to develop this study. All the seeds were obtained from the wheat germplasm bank of the Instituto Agronômico (IAC). The cultivars BH 1146 (resistant to aluminum toxicity) and Anahuac (sensitive to aluminum toxicity) belong to the species Triticum aestivum, and the genotypes P33, P19 (both resistant to aluminum toxicity), and IAC 1003 (sensitive to aluminum toxicity) belong to the species Triticum turgidum ssp. durum. The Anahuac and IAC 1003 cultivars were used as controls, as they are sensitive to aluminum toxicity.

The genotypes, P19 and P33 come from an interspecific cross (Triticum aestivum X Triticum turgidum ssp. durum), and the cv BH 1146, is the donor of the ALMT gene. Therefore, the gene that confers resistance to aluminum toxicity on P19 and P33 comes from the cv BH 1146 (Silva et al., 2010). The cv IAC 1003 was used in backcrossing, which gave rise to the genotypes for recovering the agronomic traits of durum wheat. 


\section{Preparation of the metaphase chromosomes}

Pretreatment, fixation, and preparation of the metaphase chromosomes were made ready following the method of Kirov et al. (2014), modified by Oliveira and Pinto-Maglio (2017).

\section{Selection of primers for amplification of the ALMT1 gene}

The base pair sequences of the ALMT1 gene were retrieved from the National Center for Biotechnology Information - NCBI site (www.ncbi.nlm.nih.gov). The sequence of $T$. aestivum, cV ET8, was selected, which has the complete coding sequence [Coding DNA Sequence - CDS (gi/71067632/gb/DQ072260.1)]. Various primer pairs were designed from this sequence, and the primer pairs ALMT1-ET8-1 (F: GTGCATGGTTAAGTACCCTTTG and R: ACGAATCTTTGTCATGGAATGC, annealing temperature of $60^{\circ} \mathrm{C}$ ) were selected. Fragment size, in base pairs, after amplification by PCR (Polymerase Chain Reaction), was estimated by means of the Primer 3 program. For the primer ALMT1-ET8-1, a size of $525 \mathrm{bp}$ was estimated and for this estimate, the following sequences of the ALMT1 gene available on the NCBI were used as a mold: Triticum aestivum cultivar ET8, ALMT1 gene, complete CDS (DQ072260); Triticum aestivum cultivar Tasman, ALMT1 gene, complete CDS (DQ072270.1); Triticum aestivum cultivar Cranbrook, ALMT1 gene, complete CDS (DQ072263.1); and Aegilops tauschii ecotype AUS18913, ALMT1 gene, complete CDS (DQ072271.1).

\section{Planting and collection of material for DNA extraction}

The seeds of the five genotypes germinated, with one genotype per pot. Leaves from each genotype were collected in bulk, taking approximately 20 individuals per genotype per pot at 10 days after planting. After that, the leaves were immediately frozen in liquid nitrogen and stored in a freezer $\left(-80^{\circ} \mathrm{C}\right)$ until extraction. Approximately $100 \mathrm{mg}$ of macerated tissue was transferred to $1.5 \mathrm{~mL}$ capacity tubes, which were immersed in liquid nitrogen. The DNA was extracted according to the mini-prep protocol of Doyle and Doyle (1990).

\section{Obtaining the probe of the ALMT gene region}

The DNA target sequence was amplified via PCR (Polymerase Chain Reaction) to develop the ALMT probe using the primer ALMT1-ET8-1. The concentrations adopted for a final volume of $20 \mu \mathrm{L}$ in the reactions were as follows: $2.0 \mu \mathrm{L}$ of $10 \mathrm{X}$ buffer $(500 \mathrm{mM}$ $\mathrm{KCl}) ; 1.6 \mu \mathrm{L}$ of $25 \mathrm{mM} \mathrm{MgCl}_{2} ; 2.8 \mu \mathrm{L}$ of $2.5 \mathrm{mM}$ dNTPs; $0.5 \mu \mathrm{L}$ of each primer $(1.0 \mathrm{mM}) ; 0.15 \mu \mathrm{L}$ of $5 \mathrm{U}$ of Taq DNA polymerase; and $2 \mu \mathrm{L}$ of genomic DNA /50 ng $\mu \mathrm{L}^{-1}$, completing the final volume with ultrapure water. The amplification reactions were made in the thermocycler. The following program was activated in the thermocycler (PCR): $3 \mathrm{~min}$ of denaturation at $95{ }^{\circ} \mathrm{C}$, followed by 35 amplification cycles of $95^{\circ} \mathrm{C}$ for $30 \mathrm{sec}$ (denaturation), $60^{\circ} \mathrm{C}$ for $30 \mathrm{sec}$ (annealing), $72^{\circ} \mathrm{C}$ for $30 \mathrm{sec}$ (extension), and a final extension in the last cycle at $72{ }^{\circ} \mathrm{C}$ for $10 \mathrm{~min}$. From the final volume of $20 \mu \mathrm{L}$ of the PCR reaction, $5 \mu \mathrm{L}$ was removed for confirmation of the amplification of the ALMT1 gene sequence by electrophoresis. The DNA fragments were separated by horizontal electrophoresis in $1.0 \%$ agarose gel and $0.5 \mathrm{X}$ TBE (Tris - Boric acid - EDTA) buffer at 70 volts for 1 hour 20 min and stained with the Blue Juice 6X with Gel Red mixture (a 1:1 proportion). The $1 \mathrm{~kb}$ Ladder marker was used for identifying the size of the fragments. The final product of the reaction in the thermocycler of each genotype, which exhibited amplification of the ALMT1 gene region, was purified on its own. For purification, $10 \%$ of the total volume of sodium acetate and 2.6 times the total volume of ethanol were added. The sample was inverted and kept in the freezer $\left(-20^{\circ} \mathrm{C}\right)$ for $20 \mathrm{~min}$ and then centrifuged for $20 \mathrm{~min}$ at $10,000 \mathrm{rpm}$; the supernatant was discarded. Next, $40 \mu \mathrm{L}$ of $70 \%$ ethanol was added. The tube with the sample was once more inverted and centrifuged for $5 \mathrm{~min}$ at 10,000 rpm, and the supernatant discarded. The sample was kept at ambient temperature for approximately $20 \mathrm{~min}$ for drying and then re-suspended in $7 \mu \mathrm{L}$ of ultrapure water. The material resulting from purification was dubbed the ALMT1 probe. The concentration of the ALMT1 probe after purification was evaluated in a NanoVue Plus spectrophotometer. The tubes containing the ALMT1 probes were kept at $-20{ }^{\circ} \mathrm{C}$. The concentration of the ALMT1 probe per tube, after purification, ranged from 160 to $6220 \mathrm{ng} \mu \mathrm{L}^{-1}$ between and within the genotypes. All the probes were used in in situ hybridization, and the strongest signals were observed when the concentration of the ALTM1 probe, after purification, exhibited a value in excess of $2,000 \mathrm{ng} \mu \mathrm{L}^{-1}$.

\section{Marking the ALMT1 probe}

The probe was marked with digoxigenin-11-dUTPby means of the nick translation reaction, following the manufacturer's instructions.

\section{Fluorescence in situ hybridization (FISH) of the ALMT1 probe}

The protocol based on Pendás et al. (1993) and modified as described by Iacia and Pinto-Maglio (2013) was used for applying the FISH technique to the chromosomes. The signal was detected using an antibody, the anti-digoxigenin conjugated with fluorescein isothiocyanate (FITC), and the signal was amplified using three antibodies, the anti-digoxigenin-mouse, antimouse-digoxigenin, and anti-digoxigenin conjugated with fluorescein isothiocyanate (FITC). The ALMT1 probe was preferentially, though not exclusively, used in the genotype from which the sequence of this probe was amplified. The chromosomes that revealed the signal with the ALMT1 probe were identified based on the karyotypes published in Oliveira and Pinto-Maglio (2017). 


\section{Fluorescence images}

Chromosome images were analyzed and captured using an epifluorescence microscope coupled to a computer containing imaging software. The filter used for fluorescence was for FICT (GREEN / UV - excitation at $450 \mathrm{~nm}$ and emission at $570 \mathrm{~nm}$ ). Chromosome images resulting from the application of different techniques were edited with image editing programs.

\section{Results and Discussion}

\section{Amplification of the ALMT1 gene fragment via PCR}

With amplification via $\mathrm{PCR}$, the accessions $\mathrm{BH}$ 1146, P19, P33 and Anahuac exhibited bands in the agarose gel corresponding to the primer ALMT1-ET8-1. As had been somewhat expected, the cv IAC 1003, sensitive to $\mathrm{Al}^{3+}$, did not reveal amplification of the fragment with the sequence of the ALMT1 gene. However, contrary to this aspect, the Anahuac $\mathrm{cv}$, though sensitive to aluminum toxicity, exhibited a band (Figure 1).

In the agarose gel, the size of the fragment of the amplified ALMT1 gene was approximately 750 bp (Figure 1). The number of base pairs may have been higher than that estimated by CDS alignment (525 bp) since the amplified region took both the region of exons and introns into account and the PCR reaction was induced by the genomic DNA of the wheat accessions. After amplification, the ALMT1 sequences, with $750 \mathrm{bp}$, were used as a probe in fluorescence in situ hybridization.

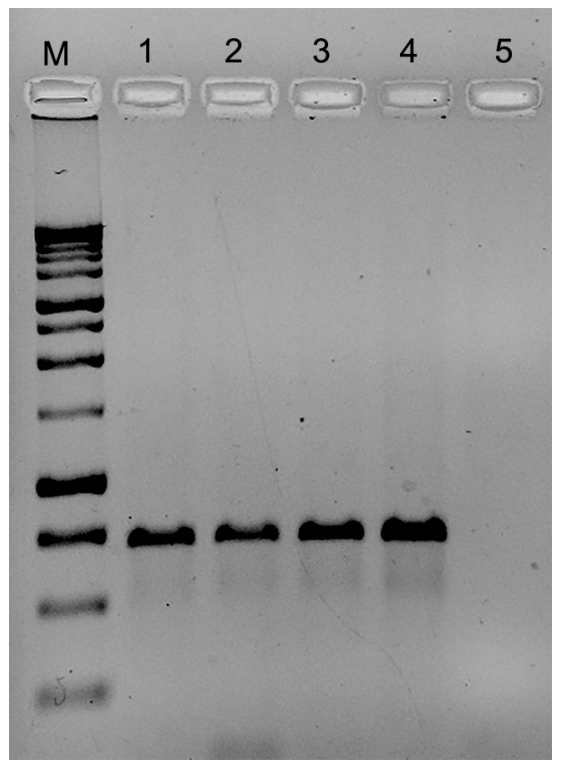

Figure 1 - Identification of the ALMT1 (aluminum-activated malate transporter) gene fragment in the genotypes 1 . Anahuac, 2. BH 1146, 3. P19, and 4. P33. Genotype 5. IAC 1003 did not reveal amplification of the ALMT1 gene fragment after amplification by PCR (Polymerase Chain Reaction). The primer pairs ALMT1-ET8-1 were used in $1.5 \%$ agarose gel. Marker: Ladder Promega $1 \mathrm{~Kb}$.

\section{Fluorescence in situ hybridization with the probe} sequence of the ALMT1 gene

The ALMT1 probe, constructed by ALMT1-ET8-1 primer, resulted in signals which were analyzed in three cells of the cv BH 1146, two cells of the cv Anahuac, five cells of the P19, and four cells of the P33 genotypes. Of the five genotypes studied, those that showed signals of hybridization with the ALMT1 probe were the Anahuac, BH 1146, P19, and P33.

The cr Anahuac, sensitive to aluminum toxicity, exhibited signals resulting from FISH-ALMT1 only in the pericentromeric region of seven chromosomes of the complement (Figure 2). The fact that the cv Anahuac (hexaploid with the ABD genome) was sensitive to aluminum toxicity and revealed the presence of the ALMT1 gene in its genome both by molecular marker and by FISH-ALMT1, suggesting that there may be a gene silencing mechanism.

The silencing of genes may result in phenotypic or physiological changes, such as expression of aluminum resistance, with no change in the DNA sequence. The possibility of gene silencing that confers resistance to aluminum toxicity is in a certain way supported by the study by Camargo et al. (2000), in which these authors, using gamma irradiation on the cv Anahuac, obtained mutant lines resistant to aluminum toxicity. Comparing the present study with the one undertaken by Camargo et al. (2000), the supposition is that gamma irradiation may have changed the formulation of the DNA structure and allowed activation of the gene that confers resistance to aluminum toxicity, i.e., the gene sequence is present but it is not expressed which would explain the presence of the ALMT1 gene fragment both through molecular markers and FISH, which was found in the Anahuac $\mathrm{cv}$ in the present study.

The cv BH 1146 showed signals in the interstitial region of the chromosomes, in the terminal region of the long arm of the chromosome and in the pericentromeric region of 12 chromosomes. Furthermore, it showed signals in the short arm of the 5D (two signals - pair 19), 6B (one signal - pair 4), and 4D (one signal - pair 20) chromosomes. Identification of the ALMT1 gene in the $4 \mathrm{D}$ chromosome ratifies location of the gene as determined by different authors on common wheat, such as Riede and Anderson (1996), Raman et al. (2005), Luo and Dvorak (1996), and Simões et al. (2012).

Riede and Anderson (1996) were not able to identify the exact locus of the aluminum tolerance gene $\left(\mathrm{Alt}_{\mathrm{BH}}\right)$ by genetic mapping in relation to the centromere or to the telomere in the 4D chromosome of the cv BH 1146. Raman et al. (2005), for their part, identified the locus of this gene in the long arm of the 4D chromosome using the Chinese Spring deletion lines at the time of QTL (quantitative trait loci) mapping of the ALMT1 gene.

For the P19 and P33 genotypes (resistant to aluminum toxicity), diverse signals were observed with 


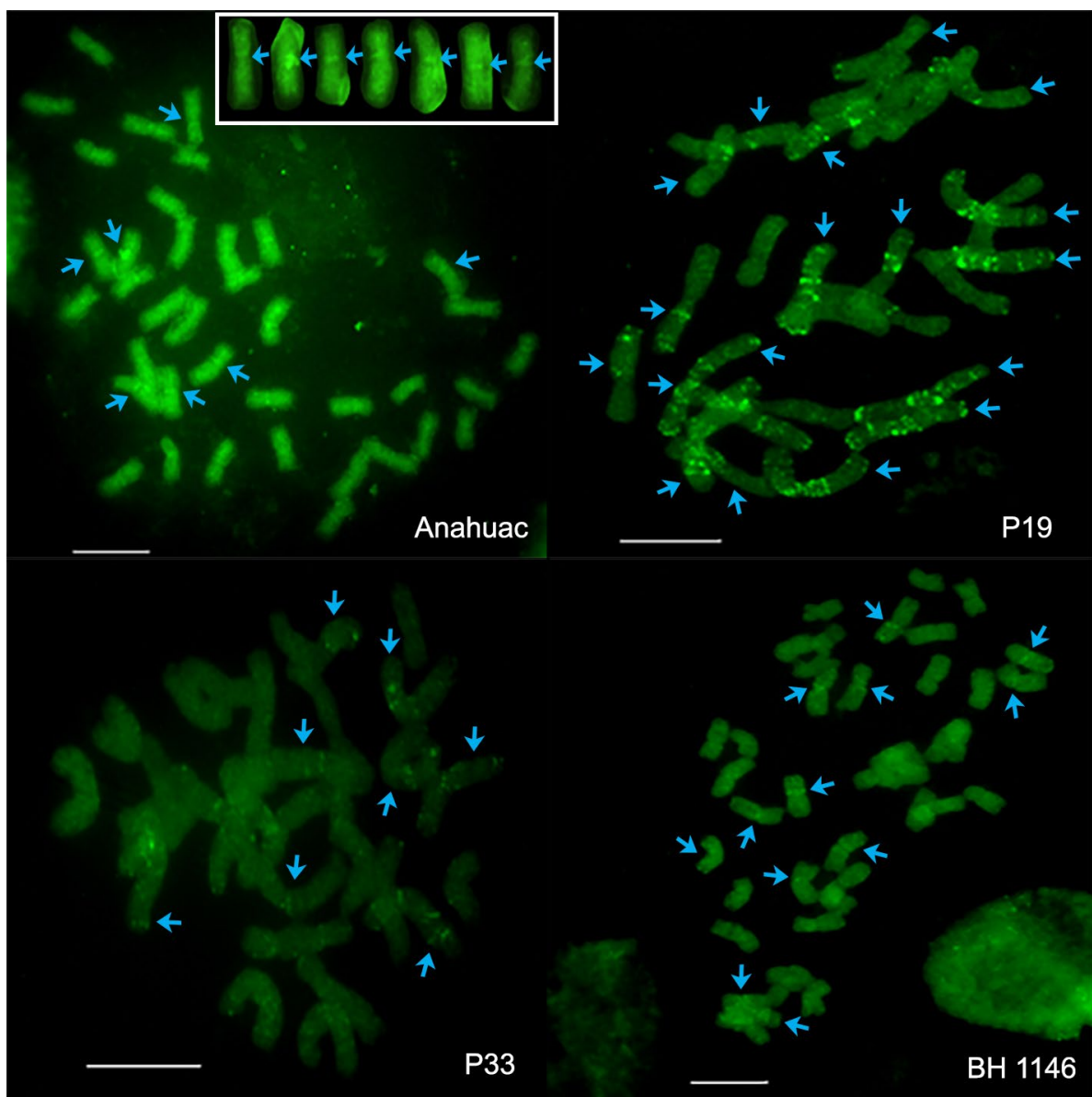

Figure 2 - Mitotic chromosomes of Triticum - Signals of the FISH (fluorescence in situ hybridization) technique with the ALMT1-ET8-1 probe in common wheat (Anahuac and BH 1146) and durum wheat (P19 and P33) genotypes. Blue arrow = chromosomes with signals. Bar $=10 \mu \mathrm{m}$.

the ALMT1 probe, in a concentrated way both in the pericentromeric regions and in the terminal and interstitial regions of the chromosomes. The diverse signals with the ALMT1 probe, dispersed in greater number and more regions in a number of chromosomes of the P19 and P33 genotypes, proved to have a different signal distribution pattern compared to the Anahuac and $\mathrm{BH} 1146$ cultivars. This mechanism seems to have occurred in a striking manner, especially in the P19 genotype, since this genotype showed approximately 45 highly evident signals (Figure 2). Thus, the ALMT1 gene in the P33 and P19 genotypes, which was transferred from the common wheat $\mathrm{BH} 1146$, may have associated with transposition elements (retrotransposons), which likely promoted both replication of this gene region and its dispersion to the other chromosomes of the complement. This observation revealed that, for unknown reason, hybridization between the cultivars promoted an increase in different copies of the gene, possibly through transposable elements (TE). An increase in the amount of TE (Ty3/gypsy) in hybrids had already been observed by Ungerer et al. (2006) in species of the Helianthus genus by qPCR.
Concentration of the ALMT1 gene fragment repeated in the genome and revealed by FISH-ALMT1 in the P19 and P33 chromosomes may be related to the increase in the total length of the chromatin (CTC) of the metaphase chromosomes. Wicker and Keller (2007) reported that the number of copies of transposable elements was related to the size of the genome of its hosts. Thus, this fact is backed up by the study of karyotyping of five wheat genotypes, which included $\mathrm{BH} 1146$, IAC 1003, Langdon, P19, and P33 (Oliveira and Pinto-Maglio, 2017), where one of the parameters estimated in the characterization of the genotypes was the calculation of the total length of the chromatin (CTC) of the metaphase chromosomes, and was precisely where higher values of CTC were found for the P19 and P3 genotypes (302.30 \pm 1.46 and $319.62 \pm 1.69$, respectively) compared to the CTC of the durum wheat cv IAC 1003 and Langdon $(246.30 \pm 1.10$ and $244.40 \pm 1.41$, respectively $)$.

A common pattern of FISH-ALMT1 signals was noted in different chromosomes in the three resistant genotypes (BH 1146, P19, and P33). Two signals were localized in the short arms of the chromosomes: 5D (pair 19) from the $\mathrm{cv} \mathrm{BH} 1146,6 \mathrm{~B}$ (pair 5) of the P33 genotype, 
and 3B (pair 1) of the P19 genotype. This common pattern of signals exhibited both repeatability and stability (Figure 3). Chromosomes were identified based on the karyotype available in Oliveira and Pinto-Maglio (2017).

From this observation, we may conclude that these two signals, related to the ALMT1 sequence and located on the short arm of the chromosomes mentioned above, were transferred from the $\mathrm{cv} \mathrm{BH} 1146$ to the P19 and P33 genotypes and that they are in fact linked to resistance to aluminum toxicity since they were identified in the three genotypes resistant to aluminum toxicity $(\mathrm{BH}$ 1146, P19, and P33) but were absent from the Anahuac and IAC 1003 cultivars (sensitive to $\mathrm{Al}^{3+}$ ).

From this knowledge of chromosome characterization (Oliveira and Pinto-Maglio, 2017) and FISH mapping of the ALMT1 gene, introduction of only the chromosome, or chromosome segment carrier of the factor that confers resistance to aluminum toxicity to durum wheat cultivars sensitive to $\mathrm{Al}^{3+}$, becomes a possiblity. This transfer can be accomplished through introgression lines, without changing the other positive agronomic traits of the durum wheat cultivars that are sensitive to $\mathrm{Al}^{3+}$, which results in savings on the number of backcrosses necessary after hybridization.

This is likely the first study involving cytogenetic mapping through FISH of the sequence that corresponds to the ALMT1 gene. Nevertheless, this type of cytogenetic analysis needs to be expanded to other wheat cultivars.

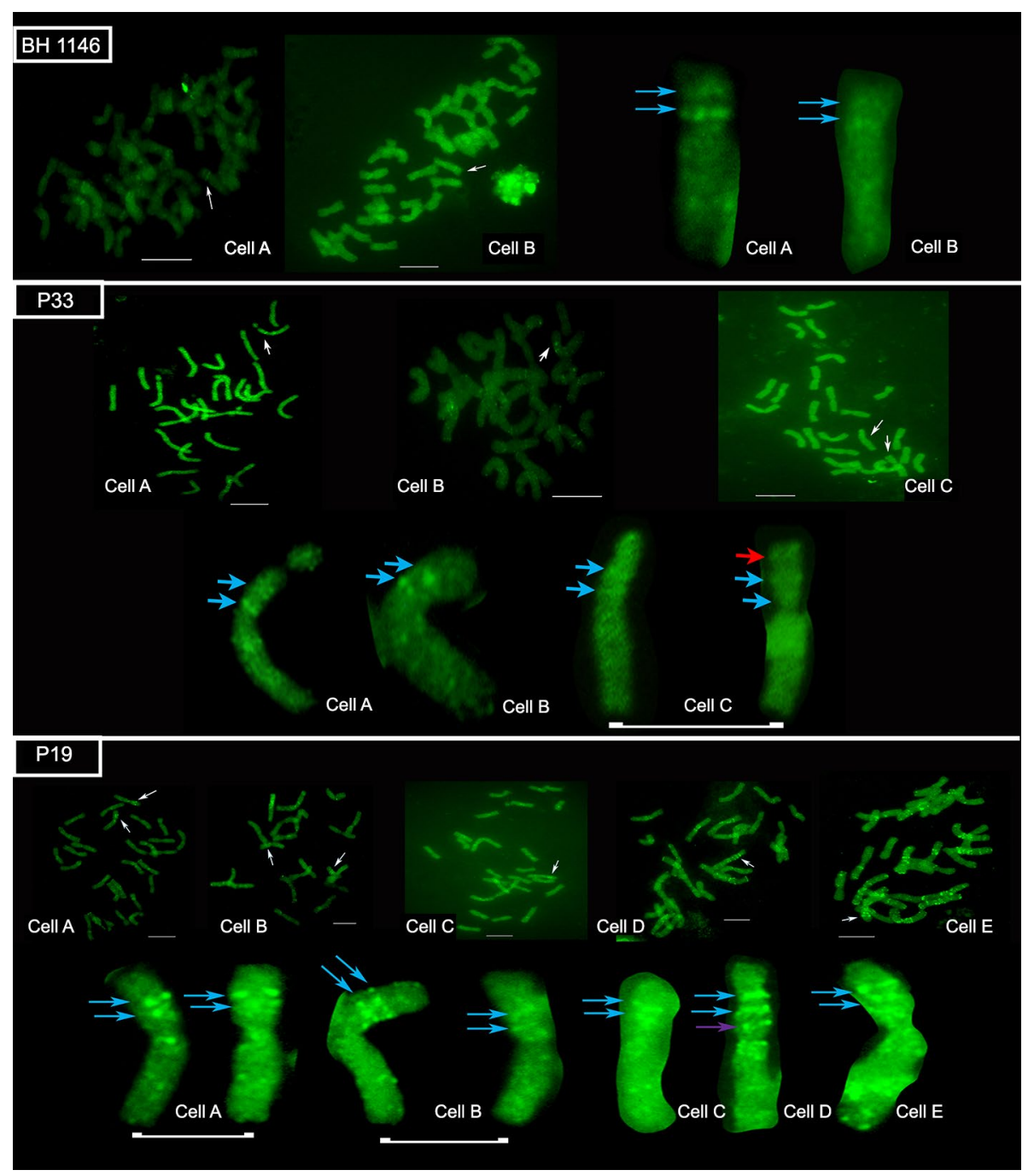

Figure 3 - Mitotic chromosomes of Triticum - Two signals of FISH-ALMT1 (fluorescence in situ hybridization - aluminum-activated malate transporter) with the ALMT1-ET8-1 probe in the short arm of the chromosomes of the genotypes BH 1146, P33, and P19. BH $1146=$ chromosomes of the A and B cells. P33 = chromosomes of the A and B cells and chromosome pair of the C cell. P19 = chromosome pair in the A and B cells; chromosome of the $C, D$, and $E$ cells. White arrow = double signal in the short arm of the chromosome. Blue arrow = double signal in the short arm of the amplified chromosome. Red arrow = signal in the satellites (SAT). Purple arrow $=$ third signal in the short arm of the chromosome. Bar $=10 \mu \mathrm{m}$. 


\section{Acknowledgments}

Our thanks to Coordenação de Aperfeiçoamento de Pessoal de Nivel Superior (CAPES) for granting a doctoral studies scholarship to the first author. We thank Dr. Carlos Augusto Colombo for the availability of the Laboratory of Molecular Biology of the Center for Plant Genetic Resources of IAC. We are grateful to Dr. Alisson Fernando Chiorato of the Center of Grains and Fibers of IAC for the availability of the wheat seeds belonging to the Germplasm Bank.

\section{Authors' Contributions}

Conceptualization: Pinto-Maglio, C.A.F.; Oliveira, E.C. Data analysis: Pinto-Maglio, C.A.F.; Oliveira, E.C. Design of Methodology: Pinto-Maglio, C.A.F.; Oliveira, E.C.; Writing and editing: Pinto-Maglio, C.A.F.; Oliveira, E.C.

\section{References}

Camargo, C.E.O.; Tulmann Neto, A.; Ferreira-Filho, A.W.P.; Felicio, J.C. 2000. Genetic control of aluminum tolerance in mutant lines of the wheat cultivar Anahuac. Euphytica 114: 47-53.

Camargo, C.E.O.; Ferreira Filho, A.W.P.; Felicio, J.C. 1999. Inheritance of aluminum tolerance in wheat. Scientia Agricola 56: 429-435 (in Portuguese, with abstract in English).

Camargo, C.E.O.; Felício, J.C.; Ferreira Filho, A.W.P.; Lobato, M.T.V. 2006. Tolerance of bread wheat, durum wheat and triticale genotypes to aluminum toxicity in nutrient solution. Bragantia 65: 43-53 (in Portuguese, with abstract in English).

Del-Guercio, A.M.F; Camargo, C.E.O. 2011. Inheritance of aluminum toxicity in durum wheat. Bragantia 70: 775-780 (in Portuguese, with abstract in English).

Doyle, J.J.; Doyle, J.L. 1990. Isolation of plant DNA fresh tissue. Focus 12: 13-15.

Iacia, A.A.S.; Pinto-Maglio, C.A.F. 2013. Mapping pachytene chromosomes of coffee using a modified protocol for fluorescence in situ hybridization. AoB Plants 5: 1-12. Doi:10.1093/aobpla/plt040.

Inostroza-Blancheteau, C.; Soto, B.; Ibáñez, C.; Ulloa, P.; Aquea, F.; Arce-Johnson, P.; Reyes-Díaz, M. 2010. Mapping aluminum tolerance loci in cereals: a tool available for crop breeding. Electronic Journal of Biotechnology 13. Doi: 10.2225/vol13issue4-fulltext-4.

Kirov, L.; Divashuk, M.; Laere, K.V.; Soloviev, A.; Khrustaleva, L. 2014. An easy "SteamDrop" method for high quality plant chromosome preparation. Molecular Cytogenetics 7: 1-10.

Lukaszewski, A.J.; Kopecky, D.; Line, G. 2012. Inversion of chromosome arms $4 \mathrm{AL}$ and $2 \mathrm{BL}$ in wheat invert the patterns of chiasma distribution. Chromosoma 121: 201-208.

Luo, M.C.; Dvorak, J. 1996. Molecular mapping of an aluminum tolerance locus on chromosome 4D of Chinese Spring wheat. Euphytica 91: 31-35.

Oliveira, É.C.; Pinto-Maglio, C.A.F. 2017. Cytomolecular characterization of cultivars and landraces of wheat tolerant and sensitive to aluminum toxicity. Bragantia 76: 456-469. Doi: http://dx.doi.org/10.1590/1678-4499.2016.278.
Pedrosa, A.; Sandal, N.; Stougaard, J.; Schweizer, D.; Bachmair, A. 2002. Chromosomal map of the model legume Lotus japonicus. Genetics 161: 1661-1672.

Pendás, A.M.; Moran, P.; Garcia-Vázquez, E. 1993. Ribosomal genes are interspersed throughout a heterochromatic arm in Atlantic salmon. Cytogenetics and Cell Genetics 63: 128-130.

Raman, H.; Zhang, K.; Cakir, M.; Appels, R.; Garvin, D.F.; Maron, L.G.; Kochian, L.V.; Moroni, J.S.; Raman, R.; Imtiaz, M.; DrakeBrockman, F.; Water, I.; Martin, P.; Sasaki, T.; Yamamoto, Y.; Matsumoto, H.; Hebb, D.M.; Delhaize, E.; Ryan, P.R. 2005. Molecular characterization and mapping of ALMT1, the aluminium-tolerance gene of bread wheat (Triticum aestivum L.). Genome 48: 781-791.

Riaz, M.; Yan, L.; Wu, X.; Hussain, S.; Aziz, O; Jiang, C. 2018. Mechanisms of organic acids and boron induced tolerance of aluminum toxicity: a review. Ecotoxicology and Environmental Safety 165: 25-35.

Riede, C.R.; Anderson, J.A. 1996. Linkage of RFLP markers to an aluminum tolerance gene in wheat. Crop Science 36: 905-909.

Sasaki, T.; Yamamoto, Y.; Ezaki, B.; Kasuara, M.; Ju Ahn, S.; Ryan, P.; Delhaize, E.; Matsumoto, H. 2004. A wheat gene encoding an aluminum-activated malate transporter. The Plant Journal 37: 645-653.

Silva, A.H.; Camargo, C.E.O.; Ramos Júnior, E.U. 2010. Potential of durum wheat genotypes for productivity and agronomic traits in São Paulo state. Bragantia 69: 535-545 (in Portuguese, with abstract in English).

Simões, C.C.; Melo, J.O.; Magalhães, J.V.; Guimarães, C.T. 2012. Genetic and molecular mechanisms of aluminum tolerance in plants. Genetics and Molecular Research 11: 1949-1957.

Soto-Cerda, B.J.; Inostroza-Blancheteau, C.; Mathías, M.; Peñaloza, E.; Zuñiga, J.; Muñoz, G.; Rengel, Z.; Salvo-Garrido, H. 2015. Marker-assisted breeding for TaALMT1, a major gene conferring aluminum tolerance to wheat. Biologia Plantarum 59: 83-91.

Tang, Z.; Li, M.; Chen, L.; Wang, Y.; Ren, Z.; Fu, S. 2014. New types of wheat chromosomal structural variations in derivatives of wheat-rye hybrids. PLoS One 9: e110282.

Ungerer, M.C.; Strakosh, S.C.; Zhen, Y. 2006. Genome expansion in three hybrid sunflower species is associated with retrotransposon proliferation. Current Biology 16: 872-873.

Wicker, T.; Keller, B. 2007. Genome-wide comparative analysis of copia retrotransposons in Triticeae, rice, and Arabidopsis reveals conserved ancient evolutionary lineages and distinct dynamics of individual copia families. Genome Research 17: 1072-1081. Doi/10.1101/gr.6214107.

Zhang, H.; Bian, Y.; Gou, X.; Zhu, B.; Xu, C.; Qi, B.; Li, N.; Rustgi, S.; Zhou, H.; Han, F.; Jiang, J.; Wettstein, D.; Liu, B. 2013. Persistent whole-chromosome aneuploidy is generally associated with nascent allohexaploid wheat. Proceedings of the National Academy of Science of the United States of America 110: 3447-3452.

Zhang, H.; Li, G.; Li, D.; Gao, D.; Zhang, J.; Yang, E.; Yang, Z. 2015. Molecular and cytogenetic characterization of new wheat - Dasypyrum breviaristatum derivatives with post-harvest re-growth habit. Genes 6: 1242-1255. 